\title{
Growth of Thin Sheet Assembled Hierarchical ZnO Nanostructures
}

\author{
Boxiang Jia,, Weina Jia, ${ }^{1}$ Jing Wang, ${ }^{1}$ Fengyu Qu, ${ }^{1}$ and Xiang Wu ${ }^{1,2}$ \\ ${ }^{1}$ Key Laboratory for Photonic and Electronic Bandgap Materials, Ministry of Education and College of Chemistry and \\ Chemical Engineering, Harbin Normal University, Harbin 150025, China \\ ${ }^{2}$ Key Laboratory of Colloid and Interface Chemistry, Ministry of Education and Department of Chemistry, Shandong University, \\ Jinan, Shandong 250100, China
}

Correspondence should be addressed to Xiang Wu, wuxiang05@gmail.com

Received 21 November 2012; Accepted 4 December 2012

Academic Editor: Xijin Xu

Copyright (C) 2012 Boxiang Jia et al. This is an open access article distributed under the Creative Commons Attribution License, which permits unrestricted use, distribution, and reproduction in any medium, provided the original work is properly cited.

Hierarchical $\mathrm{ZnO}$ nanostructures assembled from nanosheets are obtained through a facile hydrothermal route. The structure and morphology of the resultant products were characterized by X-ray diffraction (XRD), scanning electron microscope (SEM), and transmission electron microscope (TEM). The experimental results indicated that the as-synthesized ZnO nanosheets have an average thickness of approximately $50 \mathrm{~nm}$. The possible mechanism for hierarchical $\mathrm{ZnO}$ nanostructures has been discussed in detail, and it was found that sulfur powder plays a critical role in the morphology of the products. Room temperature photoluminescence property of the hierarchical $\mathrm{ZnO}$ nanostructures shows an ultraviolet emission peak at $385 \mathrm{~nm}$.

\section{Introduction}

In the past decades, enormous attention has been paid to synthesize semiconductor metal oxides due to their wide application potentials in gas sensors [1-4], photocatalysts [5-8], luminescent materials [9-12], and solar cells [13]. As one of the important semiconducting metal oxides, $\mathrm{ZnO}$ with a wide bandgap energy of $3.37 \mathrm{eV}$ has been extensively studied and applied. In these applications, it is well-known that the performance is greatly influenced by the structure and morphology of $\mathrm{ZnO}$ materials.

Therefore, various morphologies of $\mathrm{ZnO}$ materials have been synthesized, including porous nanobelts [14], flowerlike nanostructure $[15,16]$, nanotubes [17], nanowindmills [18], and hierarchical nanostructures [19-21]. However, hierarchical nanostructures have gained a great of research interest because they possess potential predominant performance. There are many reports about hierarchical $\mathrm{ZnO}$ nanostructures in the literature. Wang et al. prepared hollow $\mathrm{ZnO}$ hierarchical superstructures through a simple approach and investigated the optical properties of the $\mathrm{ZnO}$ superstructures [22]. Zhou's group synthesized hierarchically porous $\mathrm{ZnO}$ nanosheets from a hydrothermal approach [23].
Wang and his coworkers synthesized $\mathrm{ZnO}$ hierarchically porous structures with the assistance of glycine, $\mathrm{Na}_{2} \mathrm{SO}_{4}$, and polyvinyl pyrrolidone (PVP) [24]. But the above-mentioned methods need either complicated operation or the assistance of organic reagents.

In this work, we utilize a facile one-step hydrothermal route to synthesize hierarchical $\mathrm{ZnO}$ nanostructures without using any surfactants or templates. A possible growth mechanism of the as-prepared hierarchical $\mathrm{ZnO}$ products is proposed. Room temperature photoluminescence properties of the as-prepared $\mathrm{ZnO}$ products were investigated. The spectrum presents an ultraviolet emission peak at $385 \mathrm{~nm}$, revealing an excellent optical quality.

\section{Experimental Details}

The chemical reagents used in our experiment are of analytical grade without any further purification. The experiment procedures are as follows: $5 \mathrm{mmol} \mathrm{NaOH}$ and $2.5 \mathrm{mmol}$ $\mathrm{ZnSO}_{4}$ were dissolved in $5 \mathrm{~mL}$ deionized water. A little sulfur powder was dispersed into the above mixture under stirring for $15 \mathrm{~min}$ at room temperature. Then the mixed solution was transferred into autoclave and kept at $120^{\circ} \mathrm{C}$ for $10 \mathrm{~h}$. 


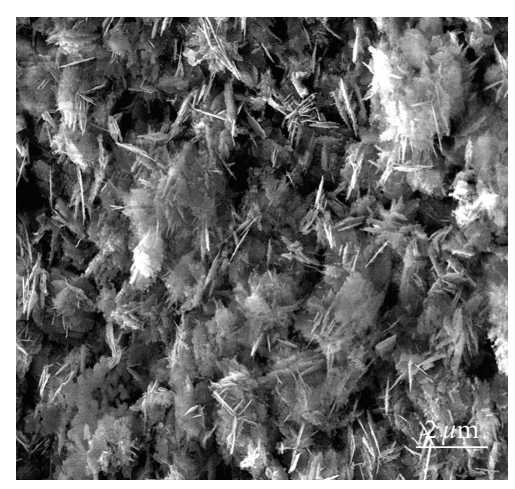

(a)

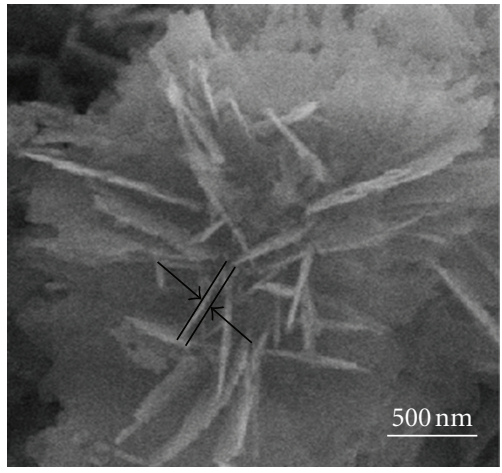

(b)

FIGURE 1: SEM images of the as-synthesized products: (a) low magnification SEM image, (b) high magnification SEM image.

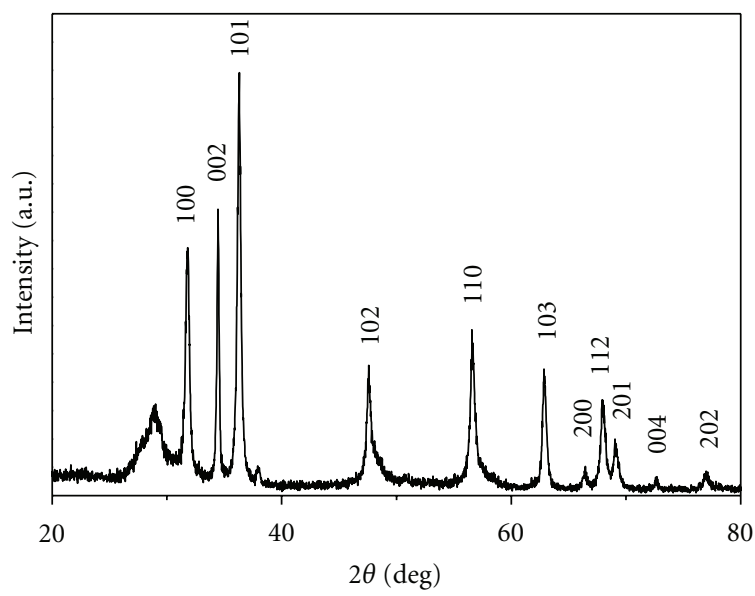

FIGURE 2: XRD pattern of the as-synthesized samples.

After the desired time, the autoclave was cooled naturally. The as-products were washed by deionized water several times and dried at $60^{\circ} \mathrm{C}$ for $5 \mathrm{~h}$.

The phase of the as-obtained products was characterized using X-ray powder diffraction (XRD, Rigaku Dmax-rB, $\mathrm{CuK} \alpha$ radiation, $\lambda=0.1542 \mathrm{~nm}, 40 \mathrm{KV}, 100 \mathrm{~mA})$. The morphology and microstructure of the samples were characterized by scanning electron microscope (SEM, Hitachi4800), transmission electron microscope (TEM, JEOL2010). Optical property of the as-synthesized hierarchical $\mathrm{ZnO}$ nanostructures was investigated by photoluminescence spectroscopy (PL SPEX FL-2T2).

\section{Results and Discussion}

Low-magnification SEM image (Figure 1(a)) presents the overall morphology of the as-synthesized samples, clearly observing that as-prepared samples consisted of many nanosheets. Further observation indicates that the thickness of nanosheets is approximately $50 \mathrm{~nm}$ as showed in Figure 1 (b), indicating that numerous nanosheets assemble into the hierarchical nanostructures.
The phase and purity of the products were investigated by X-ray diffraction pattern. A representative XRD pattern of the product is shown in Figure 2. All diffraction peaks can be well indexed to the pure wurtzite (hexagonal) structured $\mathrm{ZnO}$ (space group $\mathrm{P}_{3} \mathrm{mc}$ ) with lattice constants $a=0.3250 \mathrm{~nm}$ and $c=0.5206 \mathrm{~nm}$, consistent with the standard values reported previously for bulk $\mathrm{ZnO}$ (JCPDS Card file no. 36-1451). No other impurity peaks are detected in the as-synthesized samples, revealing the high purity of the prepared $\mathrm{ZnO}$ nanostructures. The strong and sharp diffraction peaks imply that the samples have good crystallinity.

Further morphology and structure information about the hierarchical $\mathrm{ZnO}$ nanostructures was obtained from TEM images. Figure 3(a) shows a TEM image of the hierarchical $\mathrm{ZnO}$ nanostructures obtained by ultrasonic dispersion of the as-prepared $\mathrm{ZnO}$ sample in ethanol. It is apparent that the hierarchical $\mathrm{ZnO}$ nanostructures were shattered into $\mathrm{ZnO}$ nanosheets and the nanosheets were very thin. The selected area electron diffraction (SAED) pattern (Figure 3(b)) demonstrates that the hierarchical $\mathrm{ZnO}$ nanostructures are polycrystalline in nature and the diffraction rings are indexed to (100), (002), (101) planes of $\mathrm{ZnO}$, respectively. 


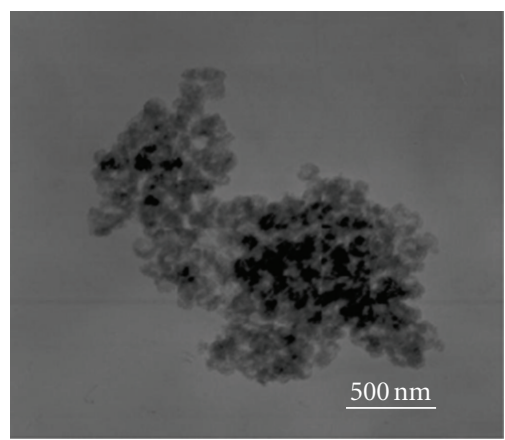

(a)

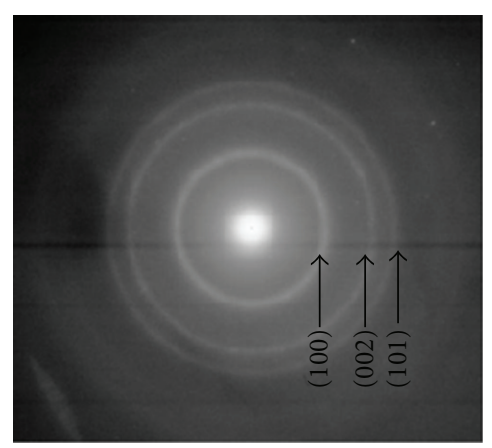

(b)

FIGURE 3: (a) TEM images and (b) SAED pattern of the as-synthesized products.

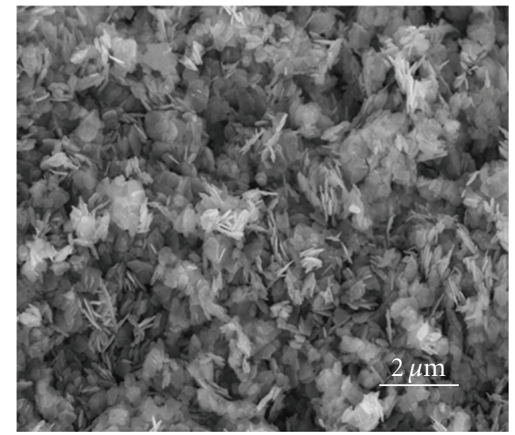

(a)

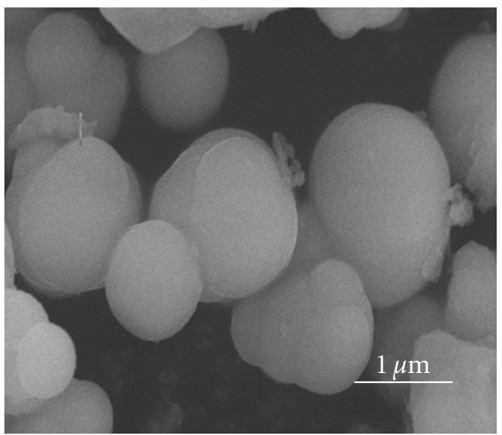

(c)

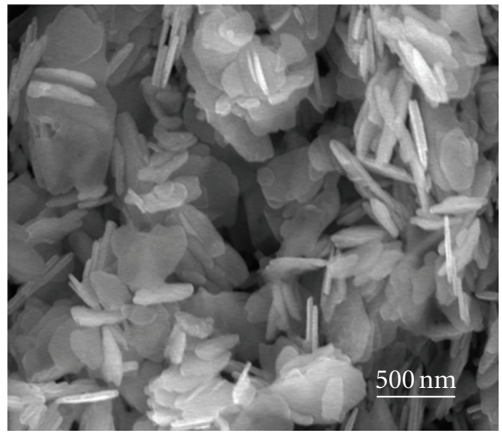

(b)

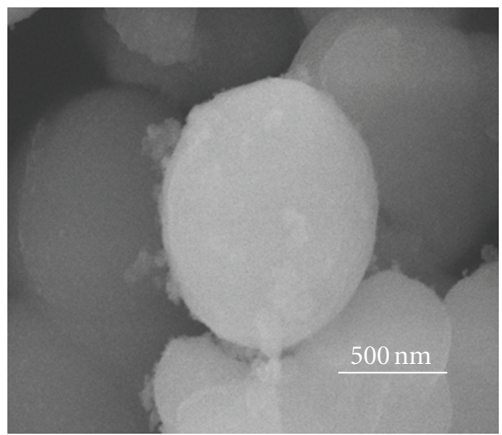

(d)

FIGURE 4: Growth control of the product morphology. SEM images of the products at different experiment parameters. (a-b) without the addition of sulfur powder, (c-d) the addition of ammonia water instead of sulfur powders.

To investigate effect of growth parameters on the morphology of hierarchical $\mathrm{ZnO}$ nanostructures, we conducted a series of comparison experiments. First, we performed the controlled experiment in the absence of sulfur powder, whereas the other experimental parameters were kept. Results showed that only the distribution of irregular thick $\mathrm{ZnO}$ nanosheets was obtained (see Figures 4(a) and 4(b)). Subsequently, we also investigated the effect of ammonia water on the morphology of the products. When $5 \mathrm{~mL}$ ammonia water instead of sulfur powder was added, hierarchical $\mathrm{ZnO}$ nanostructures did not be found (see Figures 4(c) and 4(d)), only plentiful of microspheres with a diameter of $1 \mu \mathrm{m}$ were produced. The experimental results indicate that sulfur powder plays an important role in the final morphologies of the as-synthesized product.

Based on the above experimental results, a possible growth mechanism can be proposed as follows. At first, $\mathrm{Zn}^{2+}$ reacts with $\mathrm{OH}^{-}$to form $\mathrm{Zn}(\mathrm{OH})_{2}$, then $\mathrm{Zn}(\mathrm{OH})_{2}$ can transform into $\mathrm{ZnO}$ nanosheets [25]. At the same time, sulfur powder induces $\mathrm{ZnO}$ nanosheets into self-assembly of hierarchical $\mathrm{ZnO}$ nanostructures. The overall reaction for the formation of hierarchical $\mathrm{ZnO}$ nanostructures may be simplified as follows:

$$
\begin{gathered}
\mathrm{Zn}^{2+}+2 \mathrm{OH}^{-} \longrightarrow \mathrm{Zn}(\mathrm{OH})_{2} \\
\mathrm{Zn}(\mathrm{OH})_{2} \longrightarrow \mathrm{ZnO}+\mathrm{H}_{2} \mathrm{O}
\end{gathered}
$$




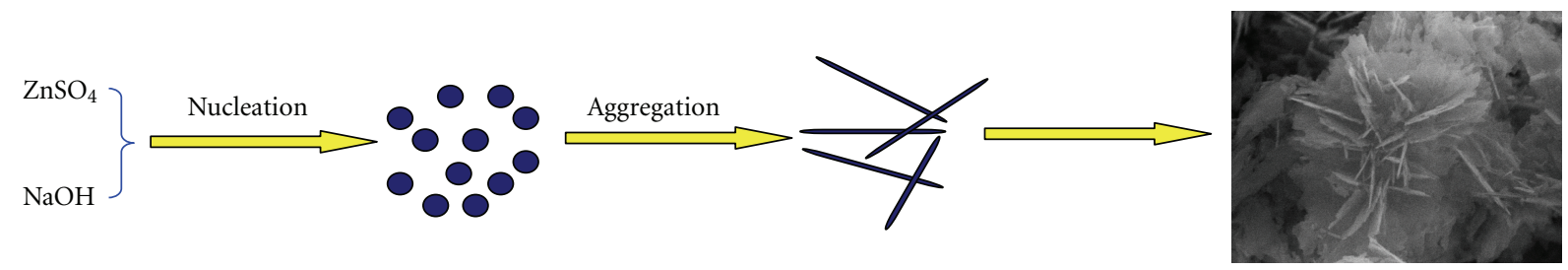

Figure 5: Schematic illustration of the possible growth mechanism of the as-synthesized hierarchical $\mathrm{ZnO}$ nanostructures.

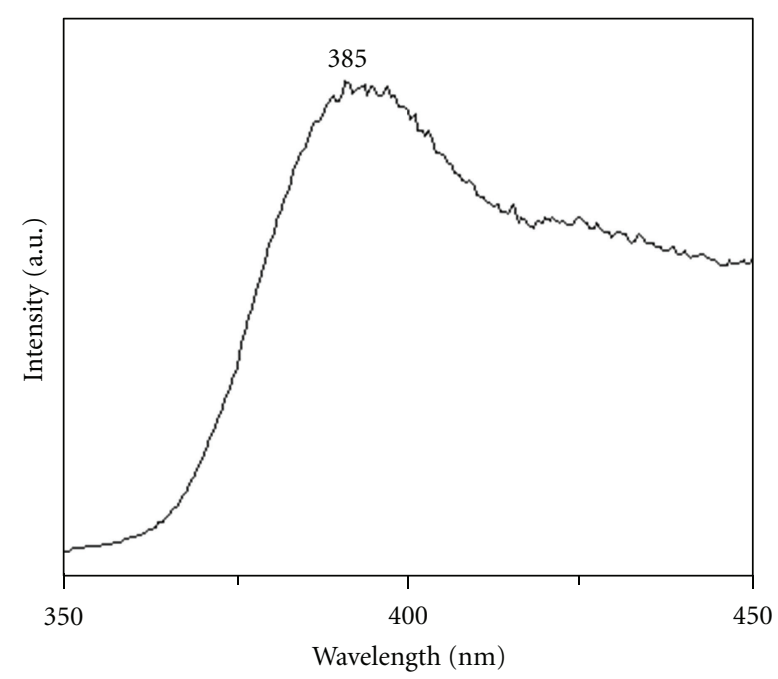

FIGURE 6: Room temperature photoluminescence spectrum of the as-synthesized hierarchical ZnO nanostructures.

Figure 5 shows the possible growth mechanism of the assynthesized hierarchical $\mathrm{ZnO}$ nanostructures.

Finally, room-temperature photoluminescence property of the as-synthesized hierarchical $\mathrm{ZnO}$ nanostructures was investigated. The experiments were conducted by $325 \mathrm{~nm}$ He-Cd laser source. Figure 6 presents a PL spectrum of the as-synthesized hierarchical $\mathrm{ZnO}$ nanostructures. The spectrum reveals an emission band located at $385 \mathrm{~nm}$, which can be commonly assigned as the near band gap exciton emission in $\mathrm{ZnO}[26,27]$. However, broad band with humps may be attributed to crystal disorder and the intrinsic defects such as oxygen vacancies, $\mathrm{Zn}$ interstitials, or impurities [28].

\section{Conclusion}

In summary, a simple hydrothermal route is developed for the fabrication of hierarchical $\mathrm{ZnO}$ nanostructures with the help of sulfur powder. The as-obtained hierarchical $\mathrm{ZnO}$ nanostructures are built up by nanosheets with the thickness of approximately $50 \mathrm{~nm}$. The TEM result indicated that the as-synthesized products possess good crystallinity. Room temperature photoluminescence spectrum demonstrates that hierarchical $\mathrm{ZnO}$ nanostructures show a broaden UV band edge emission peak at $385 \mathrm{~nm}$.

\section{Acknowledgments}

This work was sponsored by China Postdoctoral Foundation (2012M511016), Special Fund of Postdoctoral Innovation Projects in Shandong Province (201201007), Postdoctoral Initiation Foundation of Shandong University, and the Foundation for Key Project of Ministry of Education, China (no. 211046).

\section{References}

[1] F. Song, H. L. Su, J. Han, D. Zhang, and Z. X. Chen, "Fabrication and good ethanol sensing of biomorphic $\mathrm{SnO}_{2}$ with architecture hierarchy of butterfly wings," Nanotechnology, vol. 20, no. 49, Article ID 495502, 2009.

[2] L. Ge, X. Jing, J. Wang et al., "Trisodium citrate assisted synthesis of $\mathrm{ZnO}$ hollow spheres via a facile precipitation route and their application as gas sensor," Journal of Materials Chemistry, vol. 21, no. 29, pp. 10750-10754, 2011.

[3] J. Zhang, S. Wang, Y. Wang et al., "ZnO hollow spheres: preparation, characterization, and gas sensing properties," Sensors and Actuators B, vol. 139, pp. 411-417, 2009.

[4] Z. Li, Q. Zhao, W. Fan, and J. Zhan, "Porous $\mathrm{SnO}_{2}$ nanospheres as sensitive gas sensors for volatile organic compounds detection," Nanoscale, vol. 3, no. 4, pp. 1646$1652,2011$.

[5] L. L. Zhao, X. H. Ji, X. J. Sun, J. Li, W. S. Yang, and X. G. Peng, "Formation and stability of gold nanoflowers by the seeding 
approach: the effect of intraparticle ripening," The Journal of Physical Chemistry C, vol. 113, pp. 16645-16651, 2009.

[6] B. X. Jia, W. N. Jia, Y. L. Ma, X. Wu, and F. Y. Qu, " $\mathrm{SnO}_{2}$ coreshell microspheres with excellent photocatalytic properties ," Science of Advanced Materials, vol. 4, pp. 702-707, 2012.

[7] B. X. Jia, W. N. Jia, X. Wu, and F. Y. Qu, "Hierarchical porous $\mathrm{SnO}_{2}$ microflowers photocatalyst," Science of Advanced Materials, vol. 4, pp. 1127-1233, 2012.

[8] Y. Han, X. Wu, Y. Ma, L. Gong, F. Qu, and H. Fan, "Porous $\mathrm{SnO}_{2}$ nanowire bundles for photocatalyst and Li ion battery applications," CrystEngCommunity, vol. 13, no. 10, pp. 35063510, 2011.

[9] Y. H. Ni, S. Yang, J. M. Hong, L. Zhang, W. L. Wu, and Z. S. Yang, "Fabrication, characterization and properties of flowerlike $\mathrm{ZnS}$ - ZnO heterogeneous microstructures built up by ZnS-particle-strewn $\mathrm{ZnO}$ microrods," The Journal of Physical Chemistry C, vol. 112, no. 22, pp. 8200-8205, 2008.

[10] L. H. Gong, X. Wu, C. Ye, F. Y. Qu, and M. Z. An, "Aqueous phase approach to $\mathrm{ZnO}$ microspindles at low temperature," Journal of Alloys and Compounds, vol. 501, pp. 375-379, 2010.

[11] L. J. Yu, F. Y. Qu, and X. Wu, "Facile hydrothermal synthesis of novel ZnO nanocubes," Journal of Alloys and Compounds, vol. 504, pp. L1-L4, 2010.

[12] Z. Gu, M. P. Paranthaman, J. Xu, and Z. W. Pan, "Aligned ZnO nanorod arrays grown directly on zinc foils and zinc spheres by a low-temperature oxidization method," ACS Nano, vol. 3, no. 2, pp. 273-278, 2009.

[13] J. H. Qiu, M. Guo, and X. D. Wang, "Electrodeposition of hierarchical $\mathrm{ZnO}$ nanorod-nanosheet structures and their applications in dye-sensitized solar cells," ACS Applied Materials \& Interfaces, vol. 3, pp. 2358-2367, 2011.

[14] X. Cao, N. Wang, L. Wang, and L. Guo, "Porous ZnO nanobelts: synthesis, mechanism, and morphological evolutions," Journal of Nanoparticle Research, vol. 12, no. 1, pp. 143$150,2010$.

[15] J. R. Huang, Y. J. Wu, C. P. Gu et al., "Preparation of porous flower-shaped $\mathrm{SnO}_{2}$ nanostructures and their gas-sensing property," Sensors and Actuators B, vol. 147, pp. 467-474, 2010.

[16] X. Y. Xue, Z. H. Chen, L. L. Xing, C. H. Ma, Y. J. Chen, and T. H. Wang, "Enhanced optical and sensing properties of onestep synthesized $\mathrm{Pt}-\mathrm{ZnO}$ nanoflowers," The Journal of Physical Chemistry C, vol. 114, pp. 18607-18611, 2010.

[17] H. B. Chen, X. Wu, L. H. Gong, C. Ye, F. Y. Qu, and G. Z. Shen, "Hydrothermally-grown $\mathrm{ZnO}$ micro/nanotube arrays and their properties," Nanoscale Research Letters, vol. 5, pp. 570-575, 2010.

[18] L. J. Yu, F. Y. Qu, and X. Wu, "Solution synthesis and optimization of ZnO nanowindmills," Applied Surface Science, vol. 257, pp. 7432-7435, 2011.

[19] X. Wu, W. Cai, and F. Y. Qu, "Tailoring morphologies and wettability property of ZnO 1D nanostructures," Acta Physica Sinica, vol. 58, pp. 8044-8049, 2009.

[20] X. Y. Zeng, J. L. Yuan, and L. D. Zhang, "Synthesis and photoluminescent properties of rare earth doped $\mathrm{ZnO}$ hierarchical microspheres," The Journal of Physical Chemistry C, vol. 112, pp. 3503-3508, 2008.

[21] Y. Lei, F. Qu, and X. Wu, "Assembling $\mathrm{ZnO}$ nanorods into microflowers through a facile solution strategy: morphology control and cathodoluminescence properties," Nano-Micro Letters, vol. 4, no. 1, pp. 45-51, 2012.

[22] A. J. Wang, Q. C. Liao, J. J. Feng, P. P. Zhang, A. Q. Lia, and J. J. Wang, "Apple pectin-mediated green synthesis of hollow double-caged peanut-like $\mathrm{ZnO}$ hierarchical superstructures and photocatalytic applications," CrystEngComm, vol. 14, pp. 256-263.

[23] X. F. Zhou, Z. H. Hu, Y. Q. Fan, S. Chen, W. P. Ding, and N. P. Xu, "Microspheric organization of multilayered $\mathrm{ZnO}$ nanosheets with hierarchically porous structures," The Journal of Physical Chemistry C, vol. 112, pp. 11722-11728, 2008.

[24] X. Z. Wang, W. Liu, J. R. Liu et al., ACS Applied Materials \& Interfaces, vol. 4, no. 2, pp. 817-825, 2012.

[25] Y. Lei, J. Wang, F. Y. Qu, H. Li, and X. Wu, "Facile approach to $\mathrm{ZnO}$ nanorods by directly etching zinc substrate," Micro \& Nano Letters, vol. 5, pp. 485-488, 2011.

[26] M. D. Lourdes Ruiz Peralta, U. Pal, and R. Sanchez Zeferino, "Photoluminescence (PL) quenching and enhanced photocatalytic activity of Au-decorated $\mathrm{ZnO}$ nanorods fabricated through microwave-assisted chemical synthesis," ACS Applied Materials \& Interfaces, vol. 4, pp. 4807-4816, 2012.

[27] J. Song, J. Zhou, W. Wang et al., "Growth mechanism and photoluminescent properties of AlN/ZnO heterostructures," Journal of Physical Chemistry C, vol. 114, no. 24, pp. 1076110767, 2010.

[28] U. Pal, C. W. Kim, N. A. Jadhav, and Y. S. Kang, "Ultrasoundassisted synthesis of mesoporous $\mathrm{ZnO}$ nanostructures of different porosities," Journal of Physical Chemistry C, vol. 113, no. 33, pp. 14676-14680, 2009. 

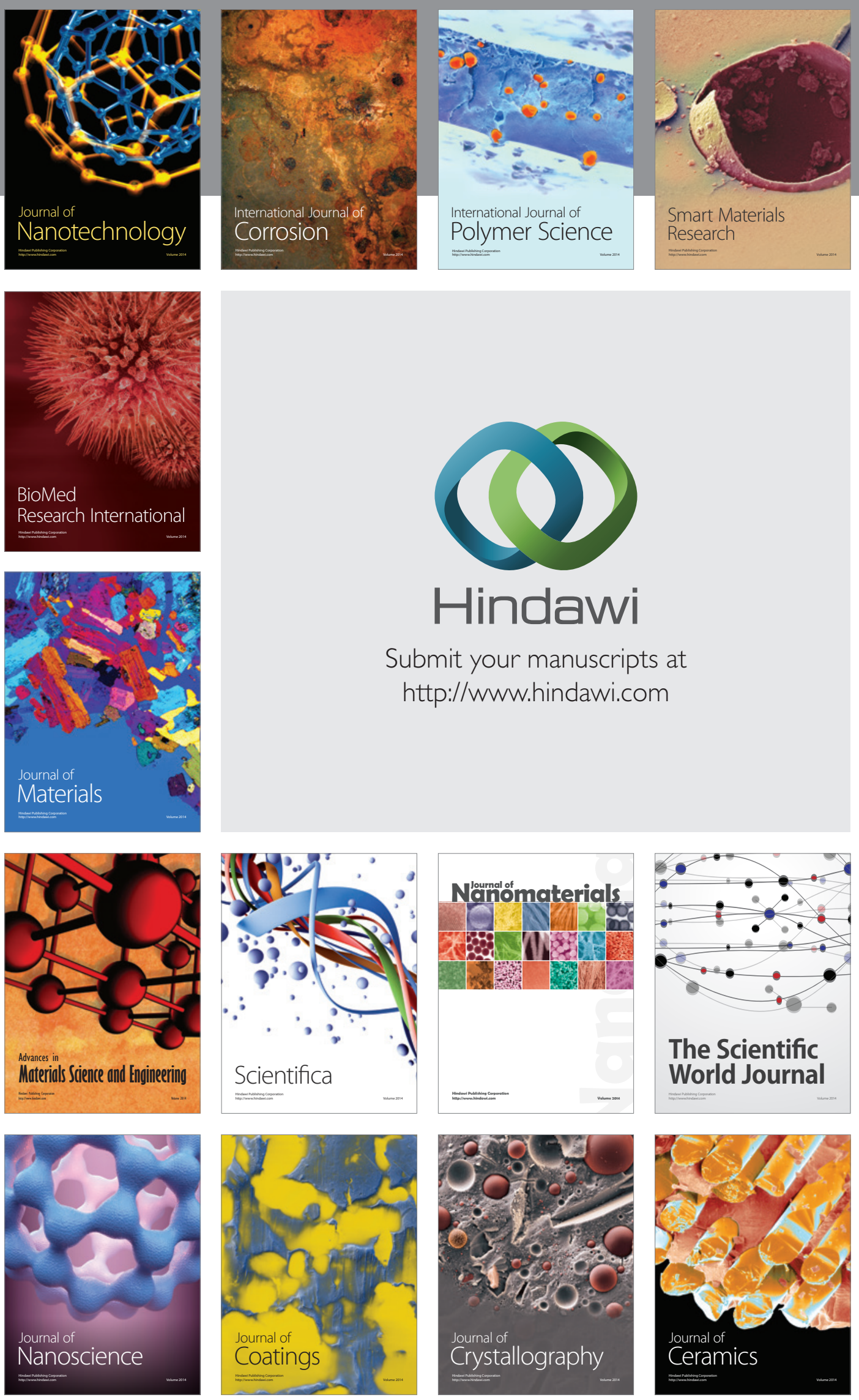

The Scientific World Journal

Submit your manuscripts at

http://www.hindawi.com

\section{World Journal}

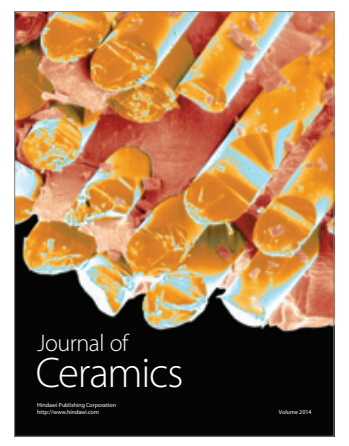

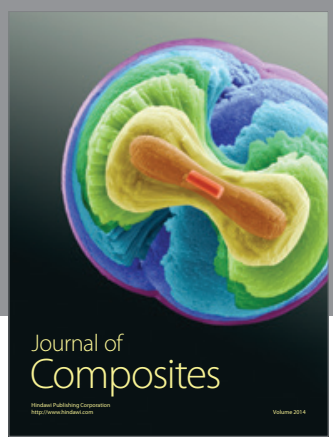
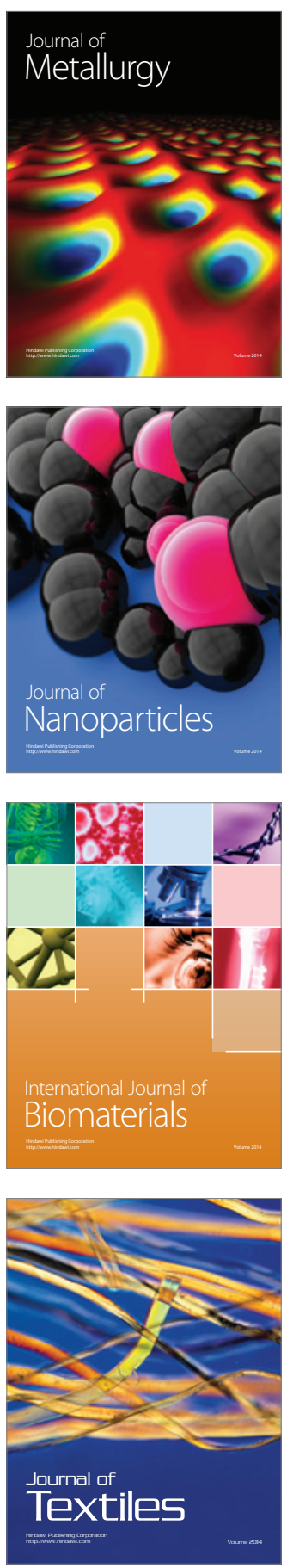